\title{
Renin-angiotensin inhibitors were associated with improving outcomes of hepatocellular carcinoma with primary hypertension after hepatectomy
}

\author{
Long-Hai Feng ${ }^{1,2 \#}$, Hui-Chuan Sun ${ }^{1,2 \#}$, Xiao-Dong Zhu ${ }^{1,2}$, Shi-Zhe Zhang ${ }^{1,2}$, Kang-Shuai Li ${ }^{1,2}$, \\ Xiao-Long $\mathrm{Li}^{1,2}$, Yan $\mathrm{Li}^{1,2}$, Zhao-You Tang ${ }^{1,2}$ \\ ${ }^{1}$ Department of Liver Surgery and Transplantation, Liver Cancer Institute and Zhongshan Hospital, Fudan University, Shanghai 200032, China; \\ ${ }^{2}$ Key Laboratory of Carcinogenesis and Cancer Invasion of Ministry of Education, Shanghai 200032, China \\ Contributions: (I) Conception and design: ZY Tang, LH Feng, HC Sun; (II) Administrative support: ZY Tang, HC Sun; (III) Provision of study \\ materials or patients: HC Sun, XD Zhu; (IV) Collection and assembly of data: LH Feng, SZ Zhang, KS Li, XL Li, Y Li; (V) Data analysis and \\ interpretation: LH Feng, HC Sun, XD Zhu; (VI) Manuscript writing: All authors; (VII) Final approval of manuscript: All authors. \\ \#These authors contributed equally to this work. \\ Correspondence to: Zhao-You Tang. Department Liver Surgery and Transplantation, Zhongshan Hospital, Fudan University, 180 Fenglin Rd, Xuhui, \\ Shanghai 200032, China. Email: zytang88@163.com.
}

Background: The activation of the renin-angiotensin system (RAS) promotes tumor progression. In this study, we aimed to assess whether RAS inhibitors (RASIs) could improve the outcome of hepatocellular carcinoma (HCC) patients with primary hypertension after curative liver resection.

Methods: Data on 387 consecutive patients with primary hypertension who underwent curative liver resection for HCC were reviewed. The study population was divided into two groups based on the type of anti-hypertensive medications: the RASI group (patients using RASIs) and the non-RASI group (patients using other anti-hypertensive drugs but not RASIs). Kaplan-Meier curves, log-rank tests and cox proportional hazards regression models were used to analyze time to recurrence (TTR) and overall survival (OS).

Results: There were 144 (37.2\%) patients in RASI group and 243 (62.8\%) in non-RASI group. The preoperative clinicopathological features were comparable between the two groups. Kaplan-Meier curves demonstrated HCC patients with RASIs had a longer TTR and OS than the patients with non-RASIs (both $\mathrm{P}<0.001)$. On multivariate analysis, RASIs administration was identified as an independent prognostic factor for TTR [hazard ratio $(\mathrm{HR})=0.52,95 \%$ confidence interval $(\mathrm{CI}), 0.38-0.70, \mathrm{P}<0.001$ ] and $\mathrm{OS}(\mathrm{HR}=0.50$, 95\% CI, 0.34-0.74, $\mathrm{P}<0.001)$. Patients in the RASI group had lower rates of extrahepatic metastases than patients in the non-RASI group (2.8\% vs. $7.8 \%, \mathrm{P}<0.042)$.

Conclusions: Targeting the RAS was associated with a reduced risk of recurrence, decreased rate of extrahepatic metastases and prolonged survival of HCC patients with primary hypertension after curative liver resection.

Keywords: Hepatocellular carcinoma (HCC); hypertension; renin-angiotensin system (RAS); prognosis

Submitted Sep 26, 2019. Accepted for publication Nov 15, 2019.

doi: $10.21037 /$ atm.2019.11.131

View this article at: http://dx.doi.org/10.21037/atm.2019.11.131 


\section{Introduction}

Hepatocellular carcinoma (HCC) is the most frequently occurring primary liver cancer which is the second leading cause of cancer-related deaths globally $(1,2)$. Unfortunately, the incidence of HCC is still expected to increase, and its global trends and predictions regarding mortality are unfavorable in the future (3). Hypertension is a common cardiovascular disease and one of the most important causes of mortality in the world (4). Moreover, hypertension is still a common comorbidity in cancer patients, common adverse reaction of cancer therapies and a predictor of efficacy for cancer treatments (5-7). In previous reports, the rates of HCC patients with hypertension varied from $14.3 \%$ to $63.7 \%$ (8-10). Regardless of whether primary hypertension or secondary hypertension is associated with tumors, it can increase cancer mortality (11). Anti-hypertensive medication needs to be more carefully studied in cancer patients.

The renin-angiotensin system (RAS) not only plays a vital role in maintaining homeostasis of blood pressure but also affects tumor biological behaviors by directly regulating tumor growth or by indirectly remodeling the tumor microenvironment $(12,13)$. In particular, angiotensin II (Ang II) and its receptor axes, the Ang II/Ang II type 1 receptor (AT1R) axis and Ang II/Ang II type 2 receptor (AT2R) axis, play an important role in tumor progression. Generally, the Ang II/AT1R axis is considered to promote tumor progression, whereas the Ang II/AT2R axis exerts opposite effects $(14,15)$. Clinically, certain tumor patients exposed to RAS inhibitors (RASIs), angiotensin-converting enzyme inhibitors (ACEIs) and Ang II type 1 receptor blockers (ARBs) may have better outcomes than patients who were not $(16,17)$. Similar beneficial effects have also been reported in HCC patients undergoing chemotherapy or radiofrequency ablation, but no surgical cases have been reported (18-20).

Furthermore, debate still exists on whether RASIs can reduce cancer risk and improve the outcomes of tumor patients (21-23). Hence, we assessed whether HCC patients with primary hypertension could benefit from the use of RASIs regarding time to recurrence (TTR) and overall survival (OS) after curative liver resection.

\section{Methods}

Between January 2010 and June 2013, 3,777 consecutive patients diagnosed with HCC underwent curative resection at Zhongshan Hospital, Fudan University (Shanghai,
China).

The inclusion criteria were as follows: (I) BCLC stages 0, A and B; (II) pathological diagnosis of HCC; (III) the minimum period between consumption of anti-hypertensive drugs and surgery was at least two weeks; (IV) Child-Pugh A or B liver function; and (V) no preoperative downstaging treatment. Patients with the following criteria were excluded: (I) recurrent HCC or combined hepatocellularcholangiocarcinoma; (II) previous medical history of hepatic or other malignant tumor resection; and (III) perioperative mortality.

The diagnostic criteria for HCC were based on the modified WHO classification of tumors of the digestive system (24). The diagnosis and treatment of primary hypertension were identified and prescribed by the patients' own cardiologists.

According to the type of anti-hypertensive drugs, the study population was divided into two groups: the RASI group, patients using RASIs, and the non-RASI, patients using other anti-hypertensive drugs except RASIs. In addition, the prognosis could be affected by the other antihypertensive drugs, Hence, more groups were established as following parallel: the beta-blocker group, patients using beta-blockers, and the non-beta-blocker, patients using other anti-hypertensive drugs except beta-blockers; the calcium channel blocker (CCB) group, patients using CCBs, and the non-CCB, patients using other anti-hypertensive drugs except CCB.

Data were extracted from medical records, updated and cross-checked and statistically analyzed. This study was approved by the Clinical Research Ethics Committee of Zhongshan Hospital, Fudan University (Shanghai, China). Written informed consent was obtained from all subjects before operation.

Patients were followed-up as in our previous report (25). TTR and OS were used as endpoints. TTR was calculated from the date of operation to the date when recurrence and/or metastasis was diagnosed. OS duration was defined as the interval between surgery and the time of death due to any cause. During the follow-up period, patients with recurrence or metastasis were treated with optimal therapeutic methods.

MedCalc statistical software (version 18.2.1) was used to analyze the data acquired from this study (26). Continuous variables were analyzed with Student's $t$ test or the MannWhitney $U$ test, and categorical variables were compared with the Chi-squared test, Fisher's exact test or Wilcoxon signed-rank test, where appropriate. Kaplan-Meier curves 


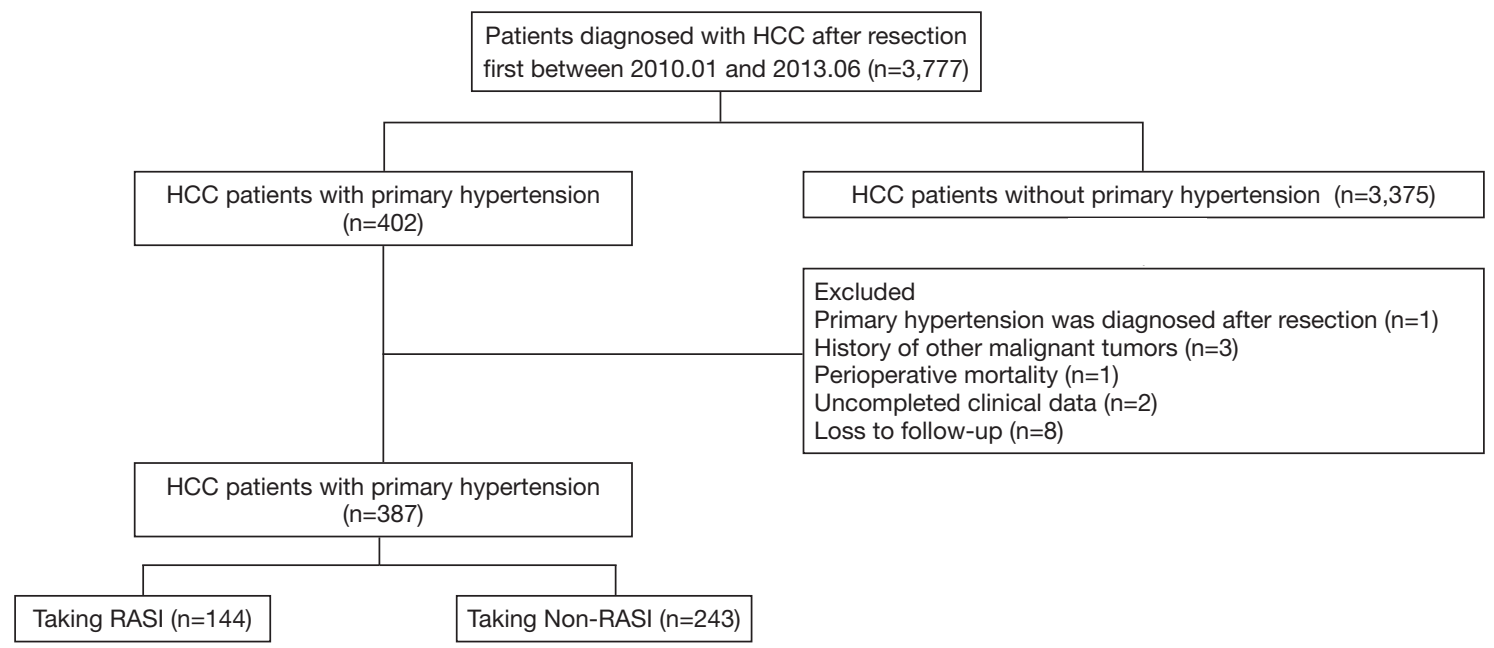

Figure 1 The flow chart of our study. HCC, hepatocellular carcinoma; RASI, the inhibitor of renin-angiotensin system.

and log-rank tests were used to analyze TTR and OS. Forest plots were generated using the forestplot package (https://CRAN.R-project.org/package=forestplot) in R version 3.5.2 (http://www.r-project.org/). All significant variables filtered by univariate analysis were entered into a Cox proportional hazards regression analysis. All statistical tests were 2 -tailed, and when the $\mathrm{P}$ value was below 0.05 , the difference was considered statistically significant.

\section{Results}

\section{Different anti-hypertensive drugs in HCC patients with primary bypertension}

Overall, 387 HCC patients with complete clinicopathological and follow-up data were included (Figure 1). According to whether the RASIs were taken or not, there were 144 patients $(37.2 \%)$ in the RASI group, 243 patients $(62.8 \%)$ in the non-RASI group; Similarly, 31 patients $(8.0 \%)$ belonged to beta-blocker group, 356 patients $(92.0 \%)$ belonged to non-beta-blocker group; 205 patients $(53.0 \%)$ belonged to CCB group and 182 patients (47.0\%) belonged to non-CCB group.

In the RASI group, 105 patients (72.9\%) took ARBs orally, and 39 patients $(27.1 \%)$ used ACEIs. In addition, 56 patients took other anti-hypertensive drugs at the same time (beta-blocker $=10, \mathrm{CCB}=53$ and Diuretic $=4$ ). In the non-RASI group, CCB $(n=152,62.6 \%)$ was the main antihypertensive drug, the second was the other antihypertensive drugs (such as reserpine, Dihydralazine and Chinese patent drugs, $n=75,30.9 \%)$ and $21(8.6 \%)$ patients took beta-blockers. The details of the anti-hypertensive drugs used are shown in Figure S1. The clinicopathological features are listed in Table 1, and no differences in baseline characteristics were found between the RASI group and the non-RASI group.

\section{Recurrence, extrahepatic metastases and survival of the study cobort}

In total, 3 patients died of non-tumor factors, including 1 patient for Parkinson's disease (RASI group); 2 patients for pulmonary infectious diseases (RASI and non-RASI group). The median follow-up duration of our study was 78.8 months $(25 \%$ quartile $=88.3 \pm 1.7$ months; $75 \%$ quartile $=68.1 \pm 0.8$ months), with the $1-, 3$ - and 5 -year recurrence rates being $15.8 \%, 36.9 \%$ and $49.6 \%$, respectively. The $1-$, 3 - and 5 -year survival rates were $95.6 \%, 83.7 \%$ and $70.3 \%$, respectively.

In the RASI group, the 1-, 3- and 5-year recurrence rates were $10.4 \%, 27.4 \%$ and $38.2 \%$, respectively, and the 1-, 3and 5 -year survival rates were $96.5 \%, 90.2 \%$ and $82.3 \%$, respectively. Correspondingly, in the non-RASI group, the 1 -, 3- and 5-year recurrence rates were $19.0 \%, 42.5 \%$ and $56.5 \%$, respectively, and the $1-, 3$ - and 5 -year survival rates were $95.1 \%, 79.8 \%$ and $64.2 \%$, respectively. Log-rank test demonstrated that the use of RASIs was significantly associated with better TTR and OS $(\mathrm{P}<0.001$ and $\mathrm{P}<0.001$, respectively; Figure 2). No differences on TTR or OS were observed in beta-blocker group $v s$. non-beta-blocker group $(\mathrm{P}=0.980$ and $\mathrm{P}=0.924$, respectively; Figure 2$)$ and 
Table 1 Clinicopathological features of hepatocellular carcinoma patients with primary hypertension

\begin{tabular}{|c|c|c|c|}
\hline Clinicopathological features & RASI (n=144) & Non-RASI ( $n=243)$ & $P$ values \\
\hline Age, range (years) & $62.3 \pm 9.2(41.0-84.0)$ & $62.1 \pm 8.3(40.0-81.0)$ & 0.886 \\
\hline Mean arterial pressure $(\mathrm{mmHg})^{*}$ & $96.7 \pm 7.0(73.3-114)$ & $96.1 \pm 7.4(70.0-113.0)$ & 0.640 \\
\hline HBsAg, positive/negative & $103 / 41(71.5 \% / 28.5 \%)$ & 175/68 (72.0\%/28.0\%) & 0.918 \\
\hline HBeAg, positive/negative & $27 / 117$ (18.8\%/81.2\%) & 38/205 (15.6\%/84.4\%) & 0.429 \\
\hline HBeAb, positive/negative & 98/46 (68.1\%/31.9\%) & 185/58 (76.1\%/23.9\%) & 0.083 \\
\hline HBcAb, positive/negative & 133/11 (92.4\%/7.6\%) & 232/11 (95.5\%/4.5\%) & 0.201 \\
\hline HCVAb, positive/negative & 2/142 (1.4\%/98.6\%) & 6/237 (2.5\%/97.5\%) & 0.470 \\
\hline ALB, range (g/L) & $40.8 \pm 3.1(31.8-51.4)$ & $40.7 \pm 3.1(32.1-53.0)$ & 0.746 \\
\hline ALT, range $(U / L)$ & $32.7 \pm 19.1(8.6-130.3)$ & $37.8 \pm 35.5(7.0-345.5)$ & 0.898 \\
\hline AST, range (U/L) & $33.3 \pm 22.8(13.0-231.0)$ & $35.8 \pm 20.2(12.0-136.0)$ & 0.153 \\
\hline ALP, range $(U / L)$ & $85.4 \pm 39.8(30.0-377.0)$ & $87.6 \pm 42.5(22.0-414.0)$ & 0.578 \\
\hline GGT, range (U/L) & $86.5 \pm 105.7(15.8-783.3)$ & $97.2 \pm 150.6(35.0-1,045.3)$ & 0.919 \\
\hline BUN, range (mmol/L) & $5.8 \pm 2.0(2.4-12.5)$ & $5.9 \pm 1.7(2.0-19.8)$ & 0.597 \\
\hline Scr, range ( $\mu \mathrm{mol} / \mathrm{L})$ & $72.5 \pm 16.9(39.0-143.6)$ & $75.3 \pm 19.3(38.4-157.5)$ & 0.137 \\
\hline PM, yes/no & $78 / 66$ (54.2\%/45.8\%) & 152/91 (62.6\%/37.4\%) & 0.104 \\
\hline Diameter, range $(\mathrm{cm})$ & $4.1 \pm 2.7(1.3-18.0)$ & $4.3 \pm 2.8(1.0-24.5)$ & 0.480 \\
\hline Single nodule, yes/no & 135/9 (93.7\%/6.3\%) & $226 / 17$ (93.0\%/7.0\%) & 0.777 \\
\hline Intact capsule, yes/no & 105/39 (72.9\%/27.1\%) & $181 / 62(74.5 \% / 25.5 \%)$ & 0.734 \\
\hline MVI, yes/no & $41 / 103(28.5 \% / 71.5 \%)$ & $69 / 174(28.4 \% / 71.6 \%)$ & 0.987 \\
\hline Differentiation, High/Moderate/Low & 8/128/8 (5.6\%/88.8\%/5.6\%) & 9/218/16 (3.7\%/89.7\%/6.6\%) & 0.648 \\
\hline Liver cirrhosis, yes/no & $77 / 67(53.5 \% / 46.5 \%)$ & $115 / 128(47.3 \% / 52.7 \%)$ & 0.242 \\
\hline TNM stages, T1a/T1b/T2/T3 & 33/72/39/0 (22.9\%/50.0\%/27.1\%/0.0\%) & 40/130/72/1 (16.5\%/53.5\%/29.6\%/0.4\%) & 0.447 \\
\hline BCLC stages, 0/A/B & $31 / 111 / 2(21.5 \% / 77.1 \% / 1.4 \%)$ & $38 / 201 / 4$ (15.6\%/82.7\%/1.6\%) & 0.341 \\
\hline Chinese stages, la/lb/lla & 104/38/2 (72.2\%/26.4\%/1.4\%) & $171 / 68 / 4(71.1 \% / 27.4 \% / 1.6 \%)$ & 0.920 \\
\hline
\end{tabular}

*, mean arterial pressure $=$ (systolic pressure $+2 x$ diastolic pressure)/3. RASI, the inhibitor of Renin-angiotensin system; ALB, albumin; ALT, alanine transaminase; AST, aspartate aminotransferase; ALP, alkaline phosphatase; GGT, $\gamma$-glutamyl transpeptidase; BUN, blood urea nitrogen; Scr, serum creatinine; AFP, alpha fetal protein; PT, prothrombin time; INR, international normalized ratio; PLT, platelets; PM, Pringle maneuver; MVI, microvascular invasion; BCLC Stages, Barcelona Clinic Liver Cancer Staging. 

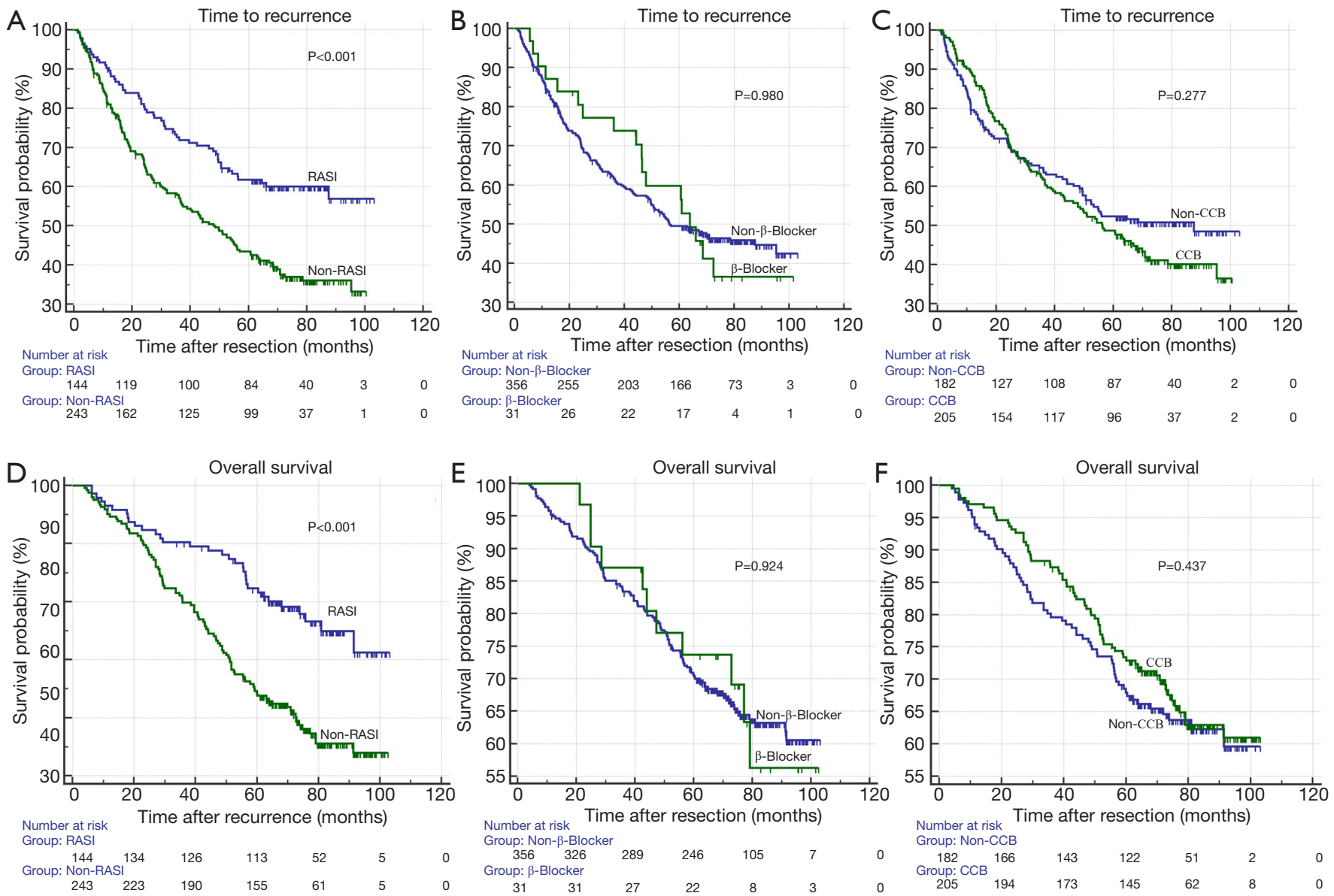

Figure 2 Tumor recurrence and overall survival of the patients taking different anti-hypertensive drugs. Recurrence of patients who taking RASI or non-RASI patients (A), $\beta$-blocker or non- $\beta$-blocker (B), and CCB or non-CCB (C); overall survival of patients who taking RASI or non-RASI patients (D), $\beta$-blocker or non- $\beta$-blocker (E), and CCB or non-CCB (F). RASI, the inhibitor of Renin-angiotensin system; CCB, calcium channel blocker.

CCB group vs. non-CCB group $(\mathrm{P}=0.277$ and $\mathrm{P}=0.437$, respectively; Figure 2).

Extrahepatic metastases occurred in 23 patients (pulmonary metastasis $=21$ and bone metastasis $=2$ ), and 4 patients were in the RASI group $(2.8 \%, 4 / 144)$ while the others were in the non-RASI group $(7.8 \%, 19 / 243$, $\mathrm{P}<0.043)$.

\section{Independent prognostic factors for TTR and OS}

The results of univariate analyses for TTR and OS are shown in Figures $S 2, S 3$. Cox proportional hazards multivariate analysis revealed that four independent factors, namely, HBeAg, alkaline phosphatase, microvascular invasion (MVI) and RASI use, were independent prognostic factors for TTR, and four independent factors, namely, albumin, aspartate aminotransferase, MVI and RASI use, were independent prognostic factors for OS (Table 2). The use of RASIs was identified as an independent prognostic factor for both TTR, with a hazard ratio (HR) of $0.52[95 \%$ confidence interval (CI), 0.38-0.70], and OS, with a HR of 0.50 (95\% CI, 0.34-0.74).

\section{TTR and OS of HCC patients using ACEIs or ARBs}

Within the RASI group, there were no differences in TTR or OS with regard to the drugs used (ACEIs vs. ARBs) $(\mathrm{P}=0.883$ and $\mathrm{P}=0.749$, respectively; Figure 3). 
Table 2 Multivariate analysis of clinicopathological parameters associated with recurrence and survival for hepatocellular carcinoma with primary hypertension

\begin{tabular}{llll}
\hline Clinicopathological parameters & $\mathrm{HR}$ & $95 \% \mathrm{Cl}$ & \\
\hline Time to recurrence & & $1.54-3.00$ & $\mathrm{P}$ values \\
HBeAg & 2.13 & $1.03-2.38$ & 0.037 \\
Alkaline phosphatase & 1.56 & $1.03-1.86$ & 0.031 \\
Microvascular invasion & 1.39 & $0.38-0.70$ & $<0.001$ \\
Use of RASls & 0.52 & & 0.003 \\
Overall survival & & $1.42-5.88$ & 0.003 \\
Albumin & 2.97 & $1.21-2.50$ & 0.004 \\
Aspartate aminotransferase & 1.74 & $1.18-2.40$ & 0.001 \\
Microvascular invasion & 1.69 & $0.34-0.74$ & \\
Use of RASls & 0.50 & & \\
\hline
\end{tabular}

RASIs, inhibitors of renin-angiotensin system; HR, hazard ratio; $\mathrm{Cl}$, confidence interval.

\section{Discussion}

Although great progress has been made in the prevention and treatment of HCC, it is still a challenging problem due to its high recurrence rate and aggressiveness $(27,28)$. In recent years, several conventional drugs, such as aspirin, beta-blockers and metformin, have become important supplemental therapies for malignant tumors (29-31). The RAS could also be a potential therapeutic target. There is still controversy regarding whether targeting the RAS can improve cancer treatment, but increasing supporting evidence has confirmed its feasibility and benefit $(15,19)$. Our data demonstrated that RASIs using was associated with a better survival for HCC patients with primary hypertension after curative liver resection.

To reduce the baseline differences within the cohort, patients with BCLC stage $0, \mathrm{~A}$ and $\mathrm{B}$ disease were included in this study. Log-rank test demonstrated that patients in the RASI group had better TTR and OS than patients in the non-RASI group (Figure 2). Cohorts from our institution demonstrated that the use of RASIs was an independent prognostic factor for TTR and OS. Lungs are the main extrahepatic organs of hematogenous metastasis of HCC. In vivo, Ang II can promote pulmonary metastasis of tumors by activating adhesion molecules in vascular endothelial cells and inhibiting tumor angiogenesis, and these effects can be attenuated by RASIs (32-34). In our study, extrahepatic metastases of HCC were observed in 23 patients, and pulmonary metastasis occurred in 21 patients.
Patients using RASIs had a lower rate of extrahepatic metastasis than patients not using RASIs.

However, the difference in prognosis could be caused by the potentially tumor-promoting effects of non-RASI use instead of the anti-tumor effects of RASI use (18). Beta-blockers were used to treat portal hypertension, and therefore could suggest an important comorbidity influencing survival $(35,36)$. Hence, more groups of antihypertensive drugs were established, but no benefits were observed in group beta-blocker $v s$. non-beta-blocker and CCB vs. non-CCB (Figure 2). However, only 31 patients $(8.0 \%, 31 / 387)$ took beta-blocker in our study and it need verifying with more cases.

Etiologically, HCC usually develops from hepatitis and fibrosis, and the Ang II/AT1R axis plays a crucial role in the pathophysiology of liver cirrhosis $(1,37)$. RASIs may delay the progression of fibrosis, ameliorate liver function and improve prognosis (18,38-40). In our study, $71.8 \%$ (278/387) of HCC patients were infected with HBV and 3 cases were complicated with HCV. Kaplan-Meier curves showed that patients using RASIs also had a lower recurrent risk and longer survival; Cox proportional hazards regression identified that the use of RASIs was significantly associated with TTR and OS in our cohort $(\mathrm{P}<0.001$ and $\mathrm{P}<0.001$, respectively; Figures S4,S5; Table S1).

Theoretically, ARBs might theoretically exert better antitumor effects than ACEIs due to its switching action on Ang II receptors. It is because of its blocking that Ang II remains mainly bound to AT2R, which is considered to 

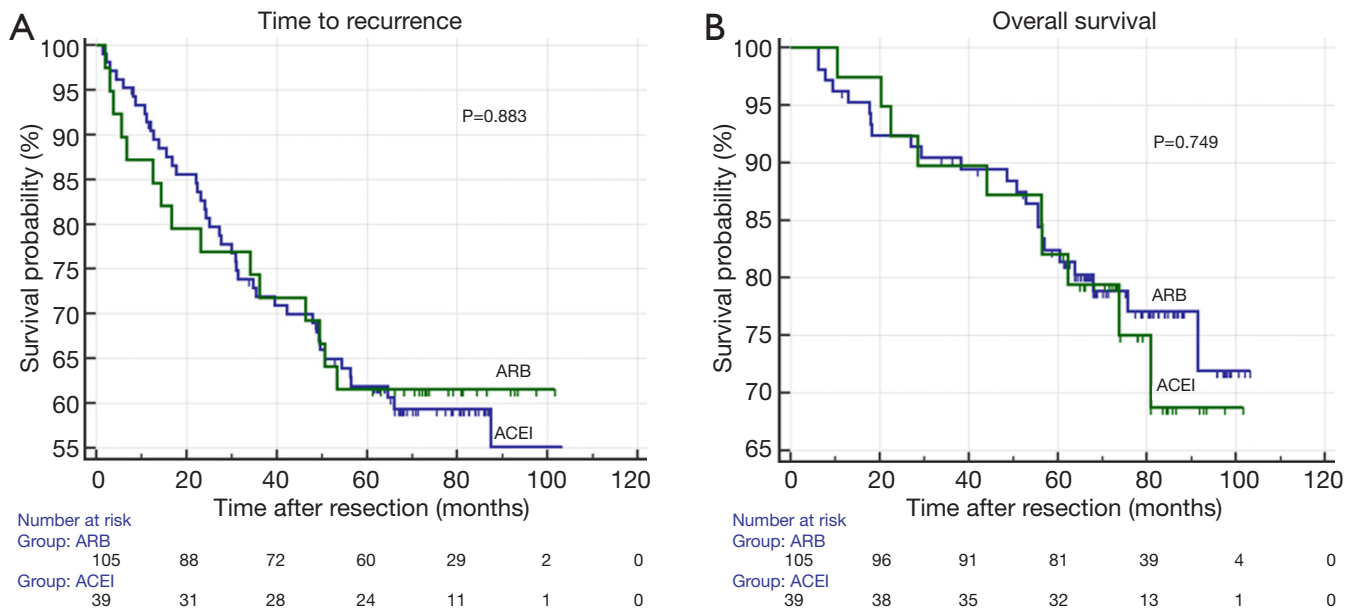

Figure 3 Recurrence and overall survival of ACEI and ARB. (A) Recurrence of ACEI and ARB patients; (B) overall survival of ACEI and ARB patients. ACEI, Angiotensin-converting enzyme inhibitors; ARB, Ang II type 1 receptor blocker.

have negative effects on tumor biological behaviors $(14,15)$. Coincidentally, a previous study not only reported that targeting the RAS could significantly prolong OS and RFS for early-stage HCC patients (BCLC stages 0 and A) after radiofrequency ablation but also demonstrated that patients using sartans (ARBs) had a better outcome than patients using ACEIs (20). However, these antitumor effects were not observed in our study (Figure 3). And some evidence has indicated that the Ang II/AT2R axis also promotes tumor progression via angiogenic and inflammatory pathways $(41,42)$. Moderate increases AT2R expression have been shown to increase instead of decrease the growth of HCC tumors and the proliferation of HCC cells in vitro and in vivo (43).

Ang II can promote VEGF expression and induce angiogenesis (44). Anti-angiogenic therapies, e.g., sorafenib and lenvatinib, are standard of care for patients of advanced HCC, and hypertension is a commonly reported adverse effect of VEGF inhibitors (7). Interestingly, the combination of RASIs with sorafenib could improve OS in patients with advanced HCC and attenuate preneoplastic lesion development in a nondiabetic rat model of steatohepatitis $(19,45)$. RASIs might be preferred Anti-hypertensive drugs for HCC patients with secondary hypertension attributed to the use of VEGF inhibitors. The RAS has significant influences on tumor progress via Ang II/AT1R axis, the Ang II/AT2R axis, the Ang [1-7]/MAS signaling pathway and other pathways, but these mechanisms have not been well explored in HCC $(12,14,46-48)$. The antitumor mechanism of RASIs has not been explored or clearly understood.
RASIs had not been used widely for HCC patients with primary hypertension. In our cohort, all patients had been identified as having primary hypertension before HCC was found, and they had stably and continuously taken antihypertensive drugs since their primary hypertension was diagnosed. On the whole, RASIs, ACEIs or ARBs, were not the top choice for HCC with primary hypertension (144 patients, $37.2 \%)$, in accordance with other reports $(18,19)$. Anti-hypertensive medication needs more careful in HCC patients.

Obviously, RASI was a kind of cardiovascular drugs instead of anticancer drugs preferentially and it might control cancer by remolding the tumor cells or reforming the tumor microenvironment instead of killing tumors directly (49). In previous clinical studies, it seemed that RASI significantly suppressed the cumulative HCC recurrence, and without prolonging patient survival (34). Probably, the beneficial effects on OS of RASI in HCC treatment could be more visible when the tumor burden was eliminated (curative resection or radiofrequency ablation) or it was combined with anticancer drugs $(19,20)$.

There are several limitations in our study. First, the main limitation was the lack of information about the time of exposure to the anti-hypertensive drugs and there were some differences on the dosage, duration and therapeutic schedule of anti-hypertensive medicine among individuals inevitably. These might affect the results partly. Second, this was a retrospective study, and all cases were collected from one hospital. A prospective study should be designed, and the antitumor effects on HCC of RASIs should be 
performed using data from multiple research centers. Third, the time of death due to any cause represents a limitation since the benefit of the treatment should be calculated on the time to death due to causes related to HCC.

Although there were some limitations that might affect the results to a certain extent, we can still conclude that targeting the RAS was associated with a reduced risk of recurrence, decreased rate of extrahepatic metastases and prolonged survival of HCC patients with primary hypertension after curative liver resection, RASIs may be preferred over other Anti-hypertensive drugs among HCC patients with hypertension, and targeting the RAS could be a supplemental therapeutic strategy for HCC.

\section{Acknowledgments}

Funding: This work was supported by the Leading Investigator Program of Shanghai municipal government (17XD1401100), the National Key Basic Research Program (973 Program; 2015CB554005) from the Ministry of Science and Technology of China, and the National Natural Science Foundation of China (81672326).

\section{Footnote}

Conflicts of Interest: The authors have no conflicts of interest to declare.

Ethical statement: The authors are accountable for all aspects of the work in ensuring that questions related to the accuracy or integrity of any part of the work are appropriately investigated and resolved. This study was approved by the Ethics Committee of Zhongshan Hospital, Fudan University (Approval No. B2012-010). Written informed consent was obtained from all subjects before operation.

\section{References}

1. Forner A, Reig M, Bruix J. Hepatocellular carcinoma. Lancet 2018;391:1301-14.

2. Torre LA, Bray F, Siegel RL, et al. Global cancer statistics, 2012. CA Cancer J Clin 2015;65:87-108.

3. Bertuccio P, Turati F, Carioli G, et al. Global trends and predictions in hepatocellular carcinoma mortality. J Hepatol 2017;67:302-9.

4. Whelton PK, Carey RM, Aronow WS, et al. 2017 ACC/ AHA/AAPA/ABC/ACPM/AGS/APhA/ASH/ASPC/
NMA/PCNA Guideline for the Prevention, Detection, Evaluation, and Management of High Blood Pressure in Adults: A Report of the American College of Cardiology/ American Heart Association Task Force on Clinical Practice Guidelines. J Am Coll Cardiol 2018;71:e127-e248.

5. Wu S, Chen JJ, Kudelka A, et al. Incidence and risk of hypertension with sorafenib in patients with cancer: a systematic review and meta-analysis. Lancet Oncol 2008;9:117-23.

6. Akutsu N, Sasaki S, Takagi H, et al. Development of hypertension within 2 weeks of initiation of sorafenib for advanced hepatocellular carcinoma is a predictor of efficacy. Int J Clin Oncol 2015;20:105-10.

7. Hamnvik OP, Choueiri TK, Turchin A, et al. Clinical risk factors for the development of hypertension in patients treated with inhibitors of the VEGF signaling pathway. Cancer 2015;121:311-9.

8. Kasmari AJ, Welch A, Liu G, et al. Independent of Cirrhosis, Hepatocellular Carcinoma Risk Is Increased with Diabetes and Metabolic Syndrome. Am J Med 2017;130:746.e741-746.e747.

9. Yu MW, Lin CL, Liu CJ, et al. Influence of Metabolic Risk Factors on Risk of Hepatocellular Carcinoma and Liver-Related Death in Men With Chronic Hepatitis B: A Large Cohort Study. Gastroenterology 2017;153:10061017.e1005.

10. Shibahara J, Ando S, Sakamoto Y, et al. Hepatocellular carcinoma with steatohepatitic features: a clinicopathological study of Japanese patients. Histopathology 2014;64:951-62.

11. Milan A, Puglisi E, Ferrari L, et al. Arterial hypertension and cancer. Int J Cancer 2014;134:2269-77.

12. Uemura H. Role of renin-angiotensin system (RAS) in cancer. Curr Cancer Drug Targets 2011;11:393.

13. Pinter M, Jain RK. Targeting the renin-angiotensin system to improve cancer treatment: Implications for immunotherapy. Sci Transl Med 2017. doi: 10.1126/ scitranslmed.aan5616.

14. Ager EI, Neo J, Christophi C. The renin-angiotensin system and malignancy. Carcinogenesis 2008;29:1675-84.

15. George AJ, Thomas WG, Hannan RD. The reninangiotensin system and cancer: old dog, new tricks. Nat Rev Cancer 2010;10:745-59.

16. Li XY, Sun JF, Hu SQ. The renin-angiotensin system blockers as adjunctive therapy for cancer: a meta-analysis of survival outcome. Eur Rev Med Pharmacol Sci 2017;21:1375-83.

17. Takagi H, Mizuno Y, Yamamoto H, et al. Effects of 
telmisartan therapy on interleukin-6 and tumor necrosis factor-alpha levels: a meta-analysis of randomized controlled trials. Hypertens Res 2013;36:368-73.

18. Kaibori M, Ishizaki M, Matsui K, et al. Evaluation of metabolic factors on the prognosis of patients undergoing resection of hepatocellular carcinoma. J Gastroenterol Hepatol 2011;26:536-43.

19. Pinter M, Weinmann A, Worns MA, et al. Use of inhibitors of the renin-angiotensin system is associated with longer survival in patients with hepatocellular carcinoma. United European Gastroenterol J 2017;5:987-96.

20. Facciorusso A, Del Prete V, Crucinio N, et al. Angiotensin receptor blockers improve survival outcomes after radiofrequency ablation in hepatocarcinoma patients. J Gastroenterol Hepatol 2015;30:1643-50.

21. Raimondi S, Botteri E, Munzone E, et al. Use of betablockers, angiotensin-converting enzyme inhibitors and angiotensin receptor blockers and breast cancer survival: Systematic review and meta-analysis. Int J Cancer 2016;139:212-9.

22. Saber S, Mahmoud A, Helal N, et al. Liver Protective Effects of Renin-Angiotensin System Inhibition Have No Survival Benefits in Hepatocellular Carcinoma Induced By Repetitive Administration of Diethylnitrosamine in Mice. Open Access Maced J Med Sci 2018;6:955-60.

23. Lee J, Lee S. Comparative Effectiveness of Combination Therapy with Statins and Angiotensin-Converting Enzyme Inhibitors versus Angiotensin II Receptor Blockers in Patients with Coronary Heart Disease: A Nationwide Population-Based Cohort Study in Korea. Pharmacotherapy 2018;38:1095-105.

24. Theise ND, Curado MP, Franceschi S, et al. Hepatocellular carcinoma In: Bosman FT, Carneiro F. editors. WHO classification of tumours of the digestive system, 4th edn. IARC: Lyon, 2010:205-16.

25. Sun HC, Zhang W, Qin LX, et al. Positive serum hepatitis $\mathrm{B}$ e antigen is associated with higher risk of early recurrence and poorer survival in patients after curative resection of hepatitis B-related hepatocellular carcinoma. J Hepatol 2007;47:684-90.

26. MedCalc Software bvba. Available online: https://www. medcalc.org. Ostend, Belgium. 2019.

27. Iñarrairaegui M, Melero I, Sangro B. Immunotherapy of Hepatocellular Carcinoma: Facts and Hopes. Clin Cancer Res 2018;24:1518-24.

28. Pinter M, Peck-Radosavljevic M. Review article: systemic treatment of hepatocellular carcinoma. Aliment Pharmacol
Ther 2018;48:598-609.

29. Li S, Dai W, Mo W, et al. By inhibiting PFKFB3, aspirin overcomes sorafenib resistance in hepatocellular carcinoma. Int J Cancer 2017;141:2571-84.

30. Nilsson MB, Sun H, Diao L, et al. Stress hormones promote EGFR inhibitor resistance in NSCLC: Implications for combinations with beta-blockers. Sci Transl Med 2017. doi: 10.1126/scitranslmed.aao4307.

31. Tseng CH. Metformin and risk of hepatocellular carcinoma in patients with type 2 diabetes. Liver Int 2018;38:2018-27.

32. Ishikane S, Hosoda H, Nojiri T, et al. Angiotensin II promotes pulmonary metastasis of melanoma through the activation of adhesion molecules in vascular endothelial cells. Biochem Pharmacol 2018;154:136-47.

33. Miyajima A, Kosaka T, Asano T, et al. Angiotensin II type I antagonist prevents pulmonary metastasis of murine renal cancer by inhibiting tumor angiogenesis. Cancer Res 2002;62:4176-9.

34. Barone M, Viggiani MT, Losurdo G, et al. Systematic review: Renin-angiotensin system inhibitors in chemoprevention of hepatocellular carcinoma. World J Gastroenterol 2019;25:2524-38.

35. Sinha R, Lockman KA, Mallawaarachchi N, et al. Carvedilol use is associated with improved survival in patients with liver cirrhosis and ascites. J Hepatol 2017;67:40-6.

36. Thiele M, Albillos A, Abazi R, et al. Non-selective betablockers may reduce risk of hepatocellular carcinoma: a meta-analysis of randomized trials. Liver Int 2015;35:2009-16.

37. Yoshiji H, Kuriyama S, Yoshii J, et al. Angiotensin-II type 1 receptor interaction is a major regulator for liver fibrosis development in rats. Hepatology 2001;34:745-50.

38. Sawada Y, Kawaratani H, Kubo T, et al. Combining probiotics and an angiotensin-II type 1 receptor blocker has beneficial effects on hepatic fibrogenesis in a rat model of nonalcoholic steatohepatitis. Hepatol Res 2019;49:284-95.

39. Yoshiji H, Noguchi R, Toyohara M, et al. Combination of vitamin $\mathrm{K} 2$ and angiotensin-converting enzyme inhibitor ameliorates cumulative recurrence of hepatocellular carcinoma. J Hepatol 2009;51:315-21.

40. Yoshiji H, Noguchi R, Yamazaki M, et al. Combined treatment of vitamin $\mathrm{K} 2$ and angiotensin-converting enzyme inhibitor ameliorates hepatic dysplastic nodule in a patient with liver cirrhosis. World J Gastroenterol 2007;13:3259-61. 
41. Pawlikowski M. Immunohistochemical detection of angiotensin receptors AT1 and AT2 in normal rat pituitary gland, estrogen-induced rat pituitary tumor and human pituitary adenomas. Folia Histochem Cytobiol 2006;44:173-7.

42. Zhang X, Lassila M, Cooper ME, et al. Retinal expression of vascular endothelial growth factor is mediated by angiotensin type 1 and type 2 receptors. Hypertension 2004;43:276-81.

43. Du H, Liang Z, Zhang Y, et al. Effects of angiotensin II type 2 receptor overexpression on the growth of hepatocellular carcinoma cells in vitro and in vivo. PLoS One 2013;8:e83754.

44. Ji Y, Wang Z, Li Z, et al. Angiotensin II induces angiogenic factors production partly via AT1/JAK2/ STAT3/SOCS3 signaling pathway in MHCC97H cells. Cell Physiol Biochem 2012;29:863-74.

45. Yoshiji H, Noguchi R, Namisaki T, et al. Combination of sorafenib and angiotensin-II receptor blocker attenuates preneoplastic lesion development in a non-diabetic rat model of steatohepatitis. J Gastroenterol 2014;49:1421-9.

46. Ishikane S, Takahashi-Yanaga F. The role of angiotensin II in cancer metastasis: Potential of renin-angiotensin system blockade as a treatment for cancer metastasis. Biochem Pharmacol 2018;151:96-103.

47. Ji Y, Wang Z, Li Z, et al. Angiotensin II Enhances Proliferation and Inflammation through AT1/PKC/NFkappaB Signaling Pathway in Hepatocellular Carcinoma Cells. Cell Physiol Biochem 2016;39:13-32.

48. Qi M, Zhou Y, Liu J, et al. AngII induces HepG2 cells to activate epithelial-mesenchymal transition. Exp Ther Med 2018;16:3471-7.

49. Tang ZY. Elimination plus Transformation-Chinese and Western Medicine Integration Brings Hope to Protracted War on Cancer. Chin J Integr Med 2018;24:563-7.

Cite this article as: Feng LH, Sun HC, Zhu XD, Zhang SZ, Li KS, Li XL, Li Y, Tang ZY. Renin-angiotensin inhibitors were associated with improving outcomes of hepatocellular carcinoma with primary hypertension after hepatectomy. Ann Transl Med 2019;7(23):739. doi: 10.21037/atm.2019.11.131 


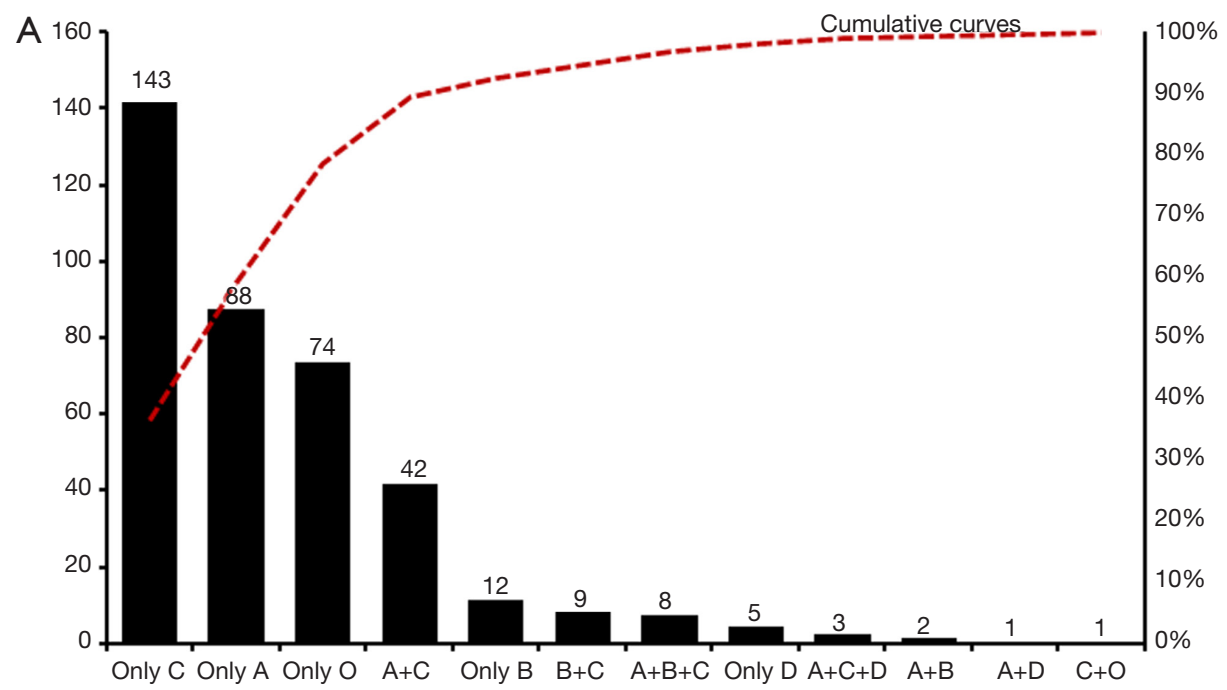

B
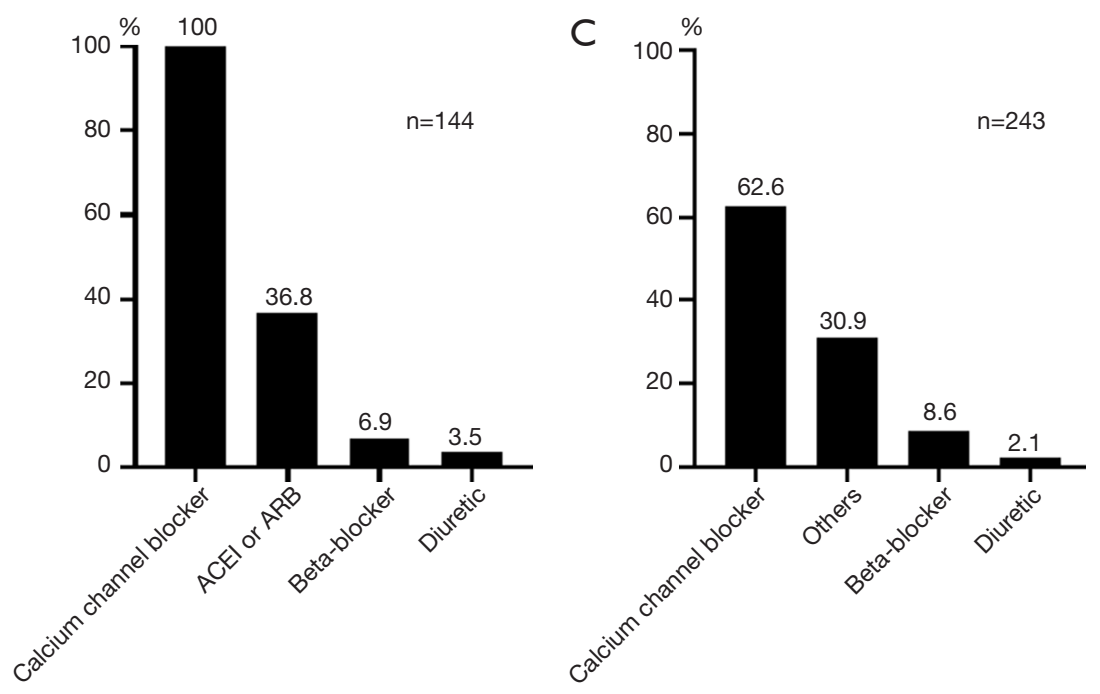

Figure S1 The details of the anti-hypertensive drugs used in our study. (A) The details of the anti-hypertensive drugs used in all cases. A, angiotensin-converting enzyme inhibitors/Ang II type 1 receptor blocker; B, beta-blocker; C, calcium channel blocker; D, diuretic; O, others, including reserpine, Dihydralazine and Chinese patent drugs. (B), the anti-hypertensive drugs used in RASI Group. All the patients took RASIs and some took more than one anti-hypertensive drugs; (C) the anti-hypertensive drugs used in non-RASI Group. Most patients took calcium channel blocker. 


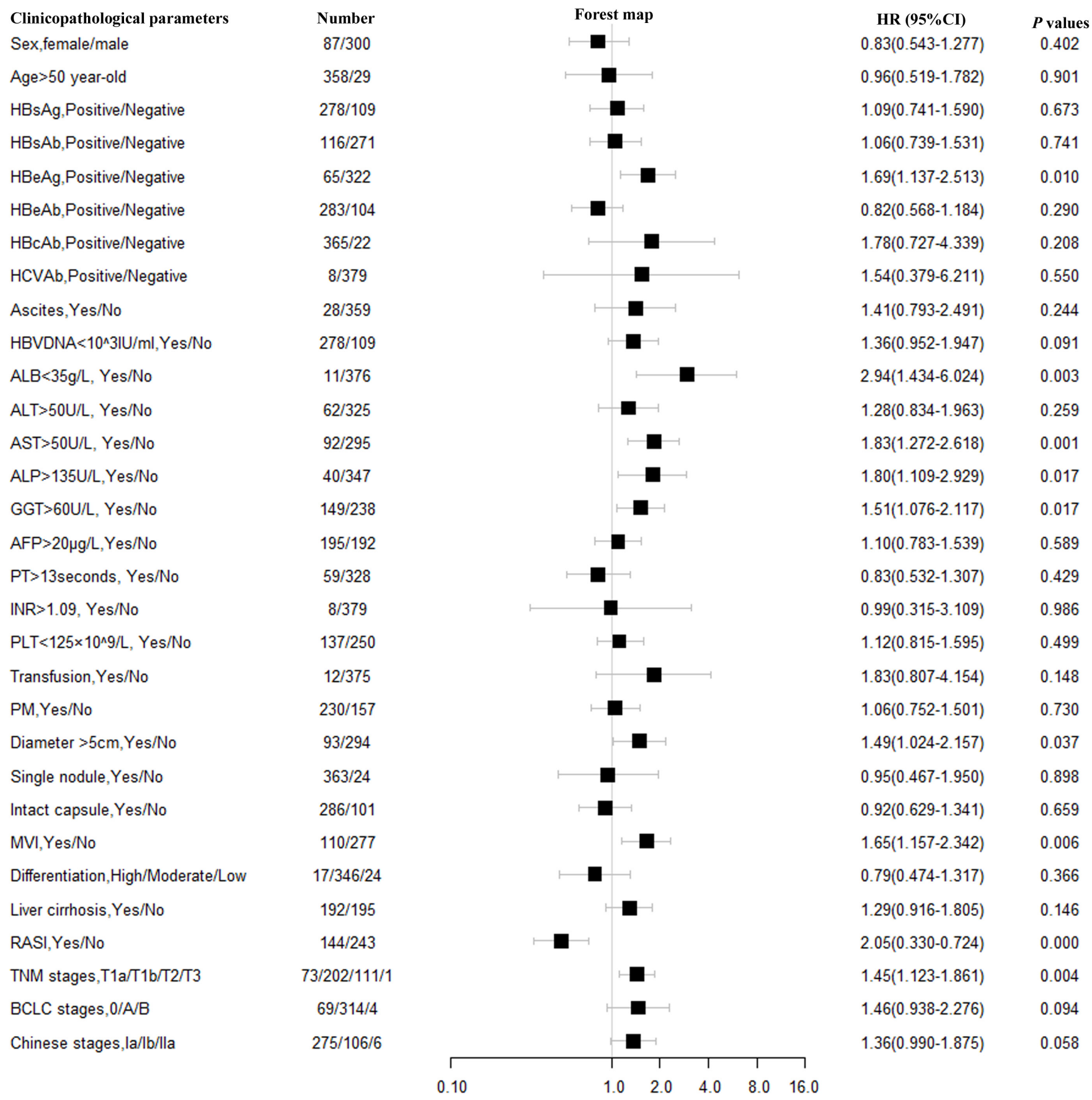

Figure S2 Forest map of univariate analysis for overall survival. ALB, albumin; ALT, alanine transaminase; AST, aspartate aminotransferase; ALP, alkaline phosphatase; GGT, $\gamma$-glutamyl transpeptidase; AFP, alpha fetal protein; PT, prothrombin time; INR, international normalized ratio; PLT, platelets; PM, Pringle maneuver; MVI, microvascular invasion; RASI, the inhibitor of Renin-angiotensin system; BCLC Stages, Barcelona Clinic Liver Cancer Staging. 


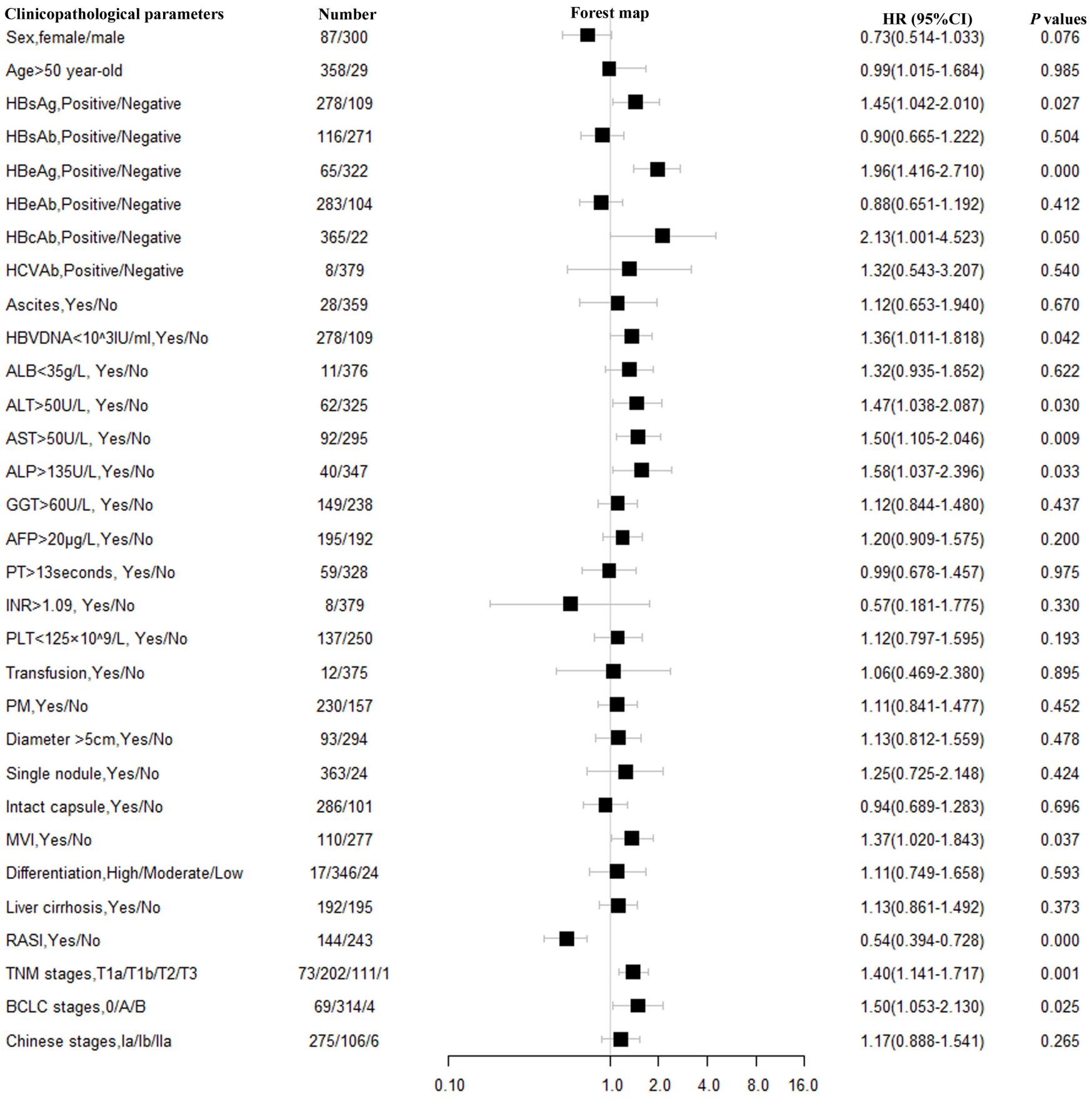

Figure S3 Forest map of univariate analysis for recurrence. ALB, albumin; ALT, alanine transaminase; AST, aspartate aminotransferase; ALP, alkaline phosphatase; GGT, $\gamma$-glutamyl transpeptidase; AFP, alpha fetal protein; PT, prothrombin time; INR, international normalized ratio; PLT, platelets; PM, Pringle maneuver; MVI, microvascular invasion; RASI, the inhibitor of Renin-angiotensin system; BCLC Stages, Barcelona Clinic Liver Cancer Staging. 


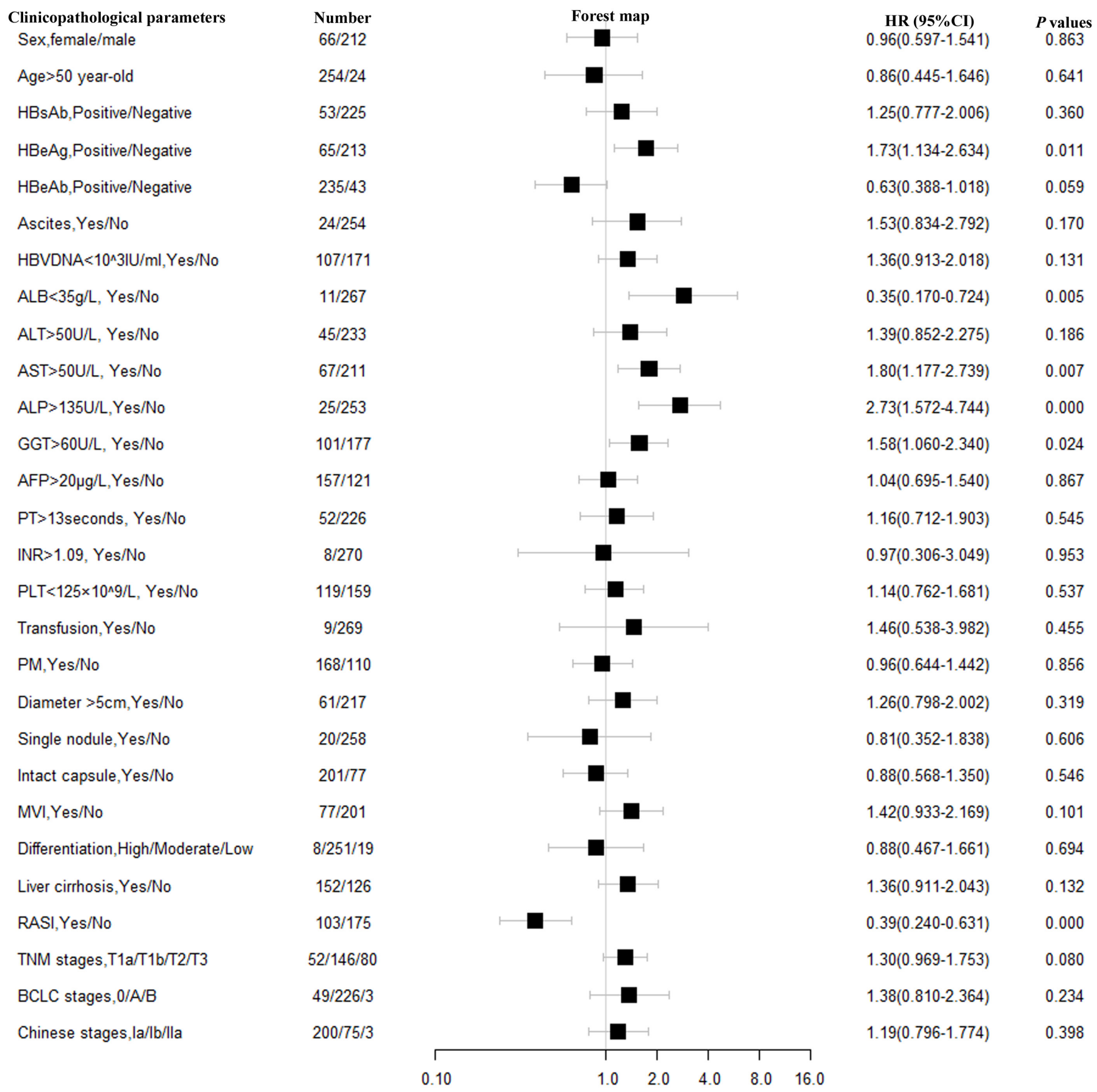

Figure S4 Forest map of univariate analysis for overall survival of HBV-hepatocellular carcinoma patients. ALB, albumin; ALT, alanine transaminase; AST, aspartate aminotransferase; ALP, alkaline phosphatase; GGT, $\gamma$-glutamyl transpeptidase; AFP, alpha fetal protein; PT, prothrombin time; INR, international normalized ratio; PLT, platelets; PM, Pringle maneuver; MVI, microvascular invasion; RASI, the inhibitor of Renin-angiotensin system; BCLC Stages, Barcelona Clinic Liver Cancer Staging. 


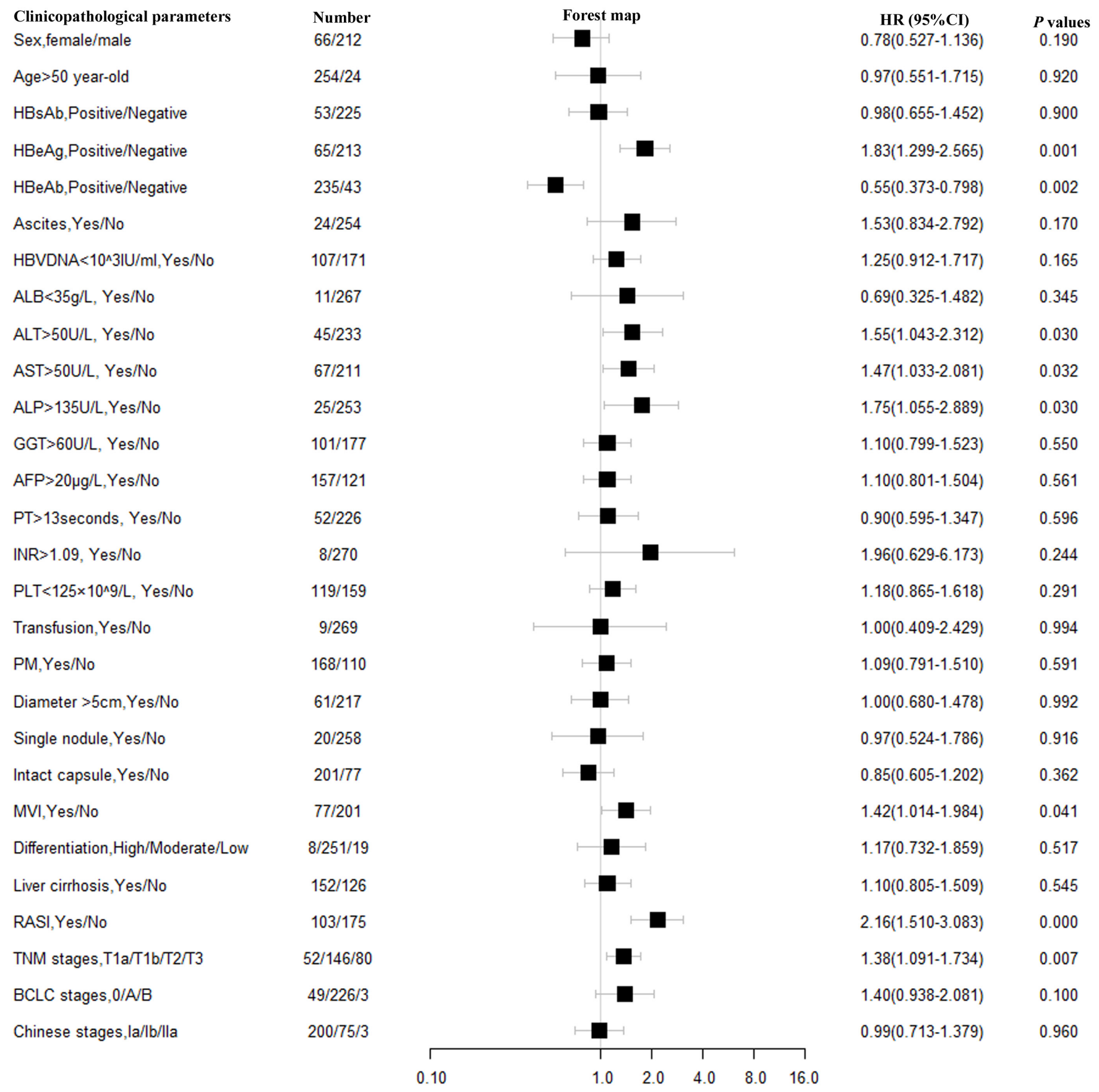

Figure S5 Forest map of univariate analysis for recurrence of HBV-hepatocellular carcinoma patients. ALB, albumin; ALT, alanine transaminase; AST, aspartate aminotransferase; ALP, alkaline phosphatase; GGT, $\gamma$-glutamyl transpeptidase; AFP, alpha fetal protein; PT, prothrombin time; INR, international normalized ratio; PLT, platelets; PM, Pringle maneuver; MVI, microvascular invasion; RASI, the inhibitor of Renin-angiotensin system; BCLC Stages, Barcelona Clinic Liver Cancer Staging. 
Table S1 Multivariate analysis of clinicopathological parameters associated with recurrence and survival for HBV-hepatocellular carcinoma with primary hypertension

\begin{tabular}{llc}
\hline Clinicopathological parameters & HR & $95 \% \mathrm{Cl}$ value \\
\hline Time to recurrence & & $1.41-2.80$ \\
HBeAg & 1.99 & $1.00-1.96$ \\
Microvascular invasion & 1.40 & $0.31-0.63$ \\
Use of RASls & 0.44 & 0.048 \\
Overall survival & & $1.09-2.57$ \\
HBeAg & 1.67 & $1.23-5.26$ \\
Albumin & 2.56 & $1.43-4.33$ \\
Alkaline phosphatase & 2.49 & 0.019 \\
Use of RASls & 0.40 & $0.24-0.64$ \\
\hline
\end{tabular}

RASIs, inhibitors of renin-angiotensin system; HR, hazard ratio; $\mathrm{Cl}$, confidence interval. 